\title{
Evaluation of Serum Level of Sclerostin in Patients with Psoriatic Arthritis
} N.E.Sorour ${ }^{1}$, N.A.Abdel hafeez ${ }^{2}$, E.M.Akl ${ }^{1}$, M.A.Mowafy ${ }^{3}$ and A.A.Mohamed ${ }^{1}$

${ }^{1}$ Dermatology, Venereology and Andrology, Dept., Faculty of Medicine, Benha Univ., Benha, Egypt

${ }^{2}$ Clinical Pathology and Immunology, Dept., Faculty of Medicine, Benha Univ., Benha, Egypt

${ }^{3}$ Rheumatology, Physical Medicine and Rehabilitation, Dept., Faculty of Medicine, Al-Azhar Univ., Cairo, Egypt. Email: Ahmed Salama @ gmail.com

Abstract

Background: Psoriatic arthritis is a chronic, immune-mediated, inflammatory arthropathy that presents with inflammation of the joints and entheses, including those of the axial skeleton, and is associated with increased mortality from cardiovascular disease. The aim of the work is to evaluate serum level of sclerostin in patients with psoriatic arthritis and to correlats its level with the severity and activity of psoriatic arthritis. Methods: This case control study included (50) subjects classified into two groups: Group I: 30 patients with psoriatic arthritis. Group II: 20 healthy age and sex matched individuals as a control group. Results: there were no statistically significant differences between case group and control group, with a mean value of $42.45 \pm 12.90$ vs $41.05 \pm 9.84$ respectively. Regarding sclerostin, it found that there were no statistically significant differences between case group and control group with a mean value of $2787.92 \pm 1838.06$ vs $2235.53 \pm 1830.67$ respectively, And it found that there were highly statistically significant differences between groups with high mean value in patients with psoriasis group of $4276.72 \pm 1239.7$ when it compared with other groups. Conclusion: The significantly higher serum sclerostin levels in PsA patients, compared with controls; there was positive significant correlation between sclerostin and each of age and PASI score. Sclerostin plays an important role in the pathogenesis of PsA and its associated bone damage, either systemic or localized.

Keywords: Sclerostin, Psoriatic, Arthritis.

\section{Introduction}

Psoriasis is a common cutaneous disorder characterized by abnormal epidermal differentiation, proliferation and inflammation mediated by dermal infiltrates, such as T cells, neutrophils, dendritic cells and macrophages. Genetics factors contribute to the initiation of psoriasis [1].

Psoriasis is a chronic skin disorder that affects approximately $2 \%$ of the population worldwide. It is characterized by well-demarcated dry plaques of variable size, covered by layers of silvery white scales. According to clinical criteria, different types of psoriatic lesions could be detected as guttate, plaque, pustular, and erythrodermic types [2].

Psoriatic arthritis (PsA) is characterized by focal bone erosions and new bone formation, suggesting an uncoupling of osteoblast-osteoclast homeostasis. It is a chronic, progressive disease that leads to significant joint damage and loss of function with negative impact on quality of life, capacity to work, and life span. Although an association between severe PsA and moderate to severe psoriasis has been reported in one series of patients with established PsA, There's little evidence of a relationship between the severity of skin and joint disease in early PsA [3].

Psoriatic arthritis occurs in $2-3 \%$ of the general population, there is a distinct increase in the prevalence of psoriatic arthritis with varied patterns of presentation that are associated with psoriasis. The genetic epidemiology of PsA also supports its unique status. PsA has been associated with human leucocyte antigen
(HLA) class 1 alleles as opposed to the class 2 alleles associated with rheumatoid arthritis (RA) [4].

Sclerostin is a 190-residue secreted glycoprotein that is predicted to contain a cysteine-knot motif and is a member of the DAN/Cerberus protein family. In patients with the rare inherited bone disorder, sclerosteosis, which is characterized by exceptionally high bone density, have been found to be homozygous for a defective sclerostin gene (SOST), and a similar high bone mass phenotype has been reported in sclerostin knock-out mice. Sclerostin null mice have very high bone mass, and conversely, severe osteopenia occurs in transgenic mice overexpressing sclerostin in osteocytes [5].

Sclerostin is secreted by osteocytes and has been shown to down-regulate the synthesis of several bone formation markers by osteogenic cells, thereby indicating the importance of sclerostin in the regulation of bone formation [6].

[7] examined the relation of serum sclerostin as one member of the wingless signal protein inhibitors to arthritic and bony manifestation of psoriasis as a model of autoimmune inflammatory arthritis. They concluded that serum sclerostin could have a significant role in the development of inflammation-associated bone damage in PsA.

The aim of the work is to evaluate serum level of sclerostin in patients with psoriatic arthritis and to correlats its level with the severity and activity of psoriatic arthritis. 


\section{Materials and methods}

This was a case control study including 60 patients and 20 control subjects had been enrolled in the study. It had been done in the Dermatology, Venereology, Andrology Outpatients Clinics and Clinical Pathology departments, Benha University Hospitals and Naser institute hospital.

This study included 80 subjects classified into two groups:

- Group I: 50 patients with psoriatic arthritis.

- Group II: 30 healthy individuals matched for age and sex were included as the control group.

A written informed consent was obtained from all subjects and approval of the Ethics committee.

\subsection{Inclusion criteria}

1- Patients age range $16-50$ years.

2- Patients with psoriatic arthritis diagnosed as CASPAR criteria which include:

- Evidence of psoriasis (current, past or family): Two points if current history of psoriasis, one point others.

- Psoriatic nail dystrophy: One point.

- Negative rheumatoid factor: One point.

- Dactylitis (current or past history): One point.

- Radiographic evidence of juxtaarticular new bone formation: One point. Three or more points have $99 \%$ specificity and $92 \%$ sensitivity for diagnosis of psoriatic arthritis.

3- Patients with psoriasis symptomatized from

- Red raised and inflamed patches of skin.

- Silver-white scales or plaques on the red patches.

- Dry skin that may crack and bleed.

- Soreness around patches.

- Itching and burning sensations around patches.

- Thick and pitted nails.

\section{Exclusion criteria:}

- Patients on steroids, disease-modifying antirheumatic drugs, or biological therapy within the last 6 months.

- Pregnant and lactating mothers.

- Other forms of inflammatory arthritis as rheumatoid arthritis and the other seronegative spondyloarthropathies.

Every patient was subjected to History taking, general examination: to exclude other causes of arthritis, Local examination: To detect psoriatic plaques and assessing severity by Psoriasis Area and Severity Index (PASI) score, And investigations:

Routine laboratory:

- Complete blood count (CBC) using automated cell counter.

- Erythrocyte sedimentation rate (ESR) in first hour estimated by Westergren method.

- Measurement of CRP using latex agglutination test.

Radiology:

- Plain X-ray on both hands postero-anterior view.

- Plain X-ray on lumbosacral spine antero-posterior and latearl views.

- Plain X-ray on both sacroiliac joints (cone) view.

\section{Serological:}

- Rheumatoid factor (IgM) titers by Enzyme-linked immunosorbent assay (ELISA). kit obtained from EUROIMMUN (Germany).

\subsection{Statistical Analysis:}

Data entry, processing and statistical analysis was carried out using Statistical package for social sciences (IBM-SPSS), version 24 (May 2016); IBM- Chicago, USA will be used for statistical data analysis. Tests of significance (Kruskal-Wallis, Wilcoxon's, Chi square, logistic regression analysis, and Spearman's correlation) were used. Data were presented and suitable analysis was done according to the type of data (parametric and nonparametric) obtained for each variable. P-values less than $0.05(5 \%)$ was considered to be statistically significant.

\section{Results}

Regarding age, there were no statistically significant differences between case group and control group, with a mean value of $42.45 \pm 12.90$ vs $41.05 \pm 9.84$ respectively.

Regarding sclerostin, it found that there were no statistically significant differences between case group and control group with a mean value of $2787.92 \pm 1838.06$ vs $2235.53 \pm 1830.67$ respectively. Table 1, And it found that there were highly statistically significant differences between groups with high mean value in patients with psoriasis group of $4276.72 \pm 1239.7$ when it compared with other groups. Figure 1

Table (1) Comparison between the studied groups according to sclerostin

\begin{tabular}{|c|c|c|c|c|c|c|}
\hline & \multicolumn{2}{|c|}{ Case group $(60)$} & \multicolumn{2}{|c|}{ Control group (20) } & \multirow[t]{2}{*}{ Statistical test (st t) } & \multirow[t]{2}{*}{ P value } \\
\hline & Mean & \pm SD & Mean & \pm SD & & \\
\hline Sclerostin & 2787.92 & 1838.06 & 2235.53 & 1830.67 & 1.17 & 0.25 \\
\hline
\end{tabular}




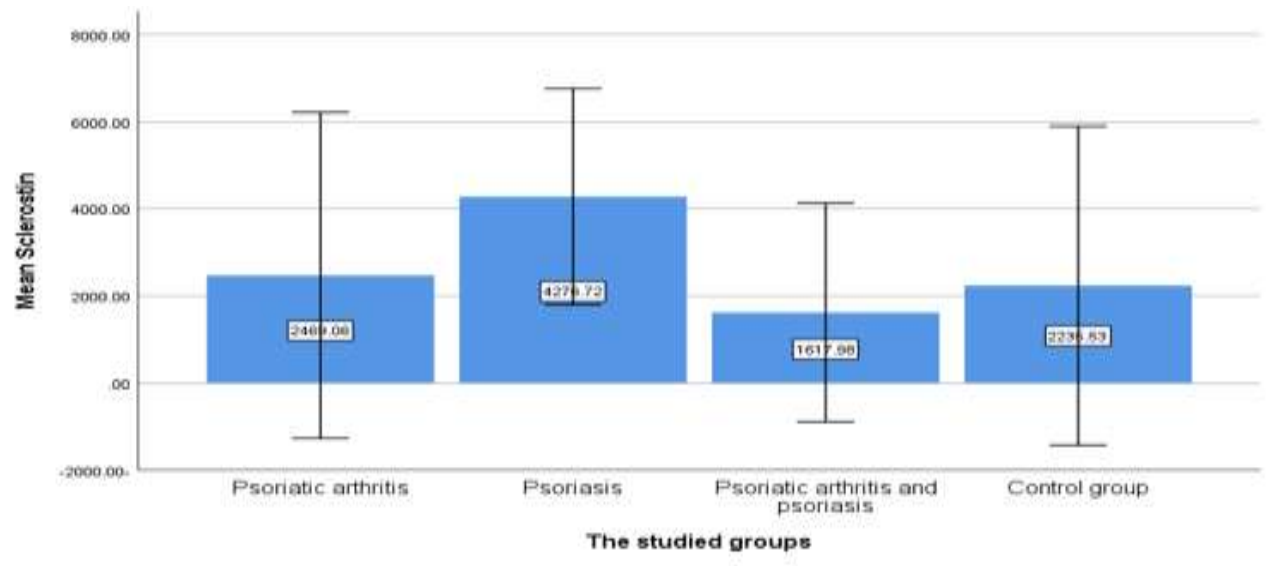

Fig. (1) Comparison between different clinical types according to sclerostin.

Figure 2, show validity of sclerostin in prediction of cases and it show that at cut off point 2180.65 the sensitivity was 50 with specificity of 60 and accuracy of 47.5 with AUC 0.593 .

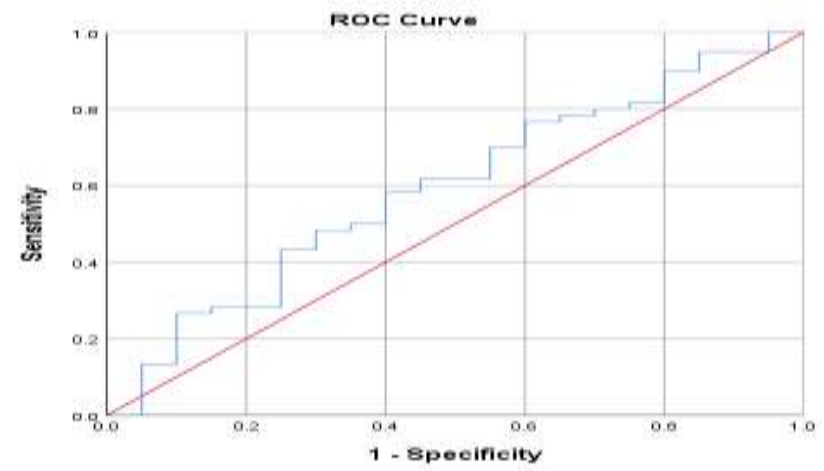

Fig. (2) Validity of sclerostin in prediction of cases.

Fig. (3), show validity of sclerostin in prediction of Psoriatic arthritis and it show that at cut off point 2113.9 the sensitivity was 50 with specificity of 50 and accuracy of 50 with AUC 0.545 .

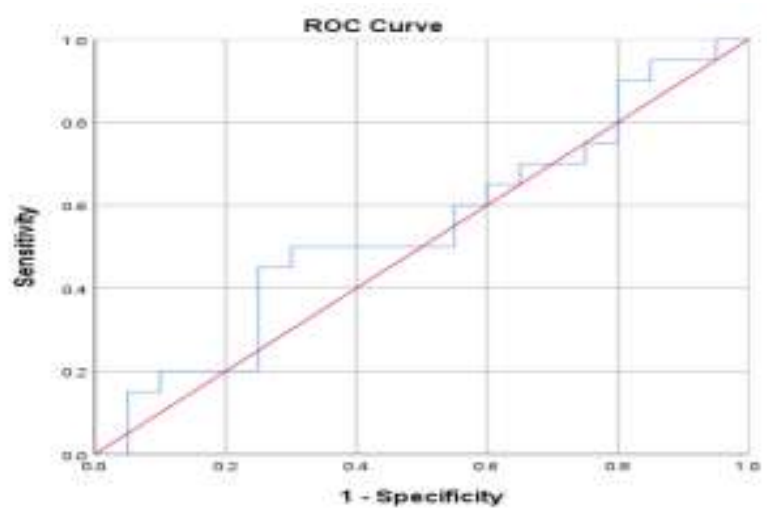

Fig. (3) Validity of sclerostin in prediction of Psoriatic arthritis.

There was a positive significant correlation between sclerostin and each of age $(\mathrm{r}=0.348, \mathrm{P}=0.007)$ and PASI score $(\mathrm{r}=0.324, \mathrm{P}=0.041)$ Table (2). 
Table (2) Correlation between sclerostin and age, duration, PASI and DAS.

\begin{tabular}{lll}
\hline Sclerostin & r & P \\
\hline Age & 0.348 & $0.007^{* *}$ \\
Duration of disease & 0.186 & 0.156 \\
PASI (40) & 0.324 & $0.041^{*}$ \\
DAS (40) & -0.07 & 0.669 \\
\hline
\end{tabular}

\section{Discussion}

Sclerostin is a monomeric glycoprotein with a cysteine knot-like domain that has homology to the Cerebrus/DAN family of bone morphogenetic protein antagonists. It is a SOST gene product expressed by osteocytes and articular chondrocytes. Because of its high conservation across vertebrate species (the amino acid sequences in the vervet, rat, and mouse are $98 \%$, $89 \%$, and $88 \%$ identical respectively to the human sequence), animal models are potentially useful for studying sclerostin. When sclerostin binds to the LRP5/6 and Frizzled coreceptors on the cell surface of osteoblasts, Wnt/ $\beta$-catenin signaling is inhibited, thereby inhibiting osteoblast differentiation, proliferation, and activity, resulting in reduced osteoblastic bone formation [5].

The present study showed that as regard sclerostin there were no statistically significant differences between case group and control group with a mean value of $2787.92 \pm 1838.06$ vs $2235.53 \pm 1830.67$ respectively. As regard different clinical types according to sclerostin, there were highly statistically significant differences between groups with high mean value in patients with psoriasis group of $4276.72 \pm 1239.7$ when it compared with other groups.

Our results were in agreement with study of Enas et al., [7] as they reported that the serum sclerostin level was significantly higher in PsA male patients compared with normal healthy age, sex, and BMI-matched controls. The findings of previous studies on rheumatoid arthritis have been controversial, as some found that the serum sclerostin level was much higher in patients compared with controls [8] and others found no significant difference in the levels between patients and controls, but this could be explained by the fact that most of the patients in that study were under medical control using methotrexate and were in a state of remission [9]. Previous studies on spondyloarthropathy patients revealed that the levels were significantly lower in patients compared with controls. However, PsA has different patterns of presentation, and owing to the fact that most of their patients presented with peripheral arthritis rather than axial, spondyloarthropathy could explain the fact that serum sclerostin was higher in patients compared with controls.

In a recent work by Tarhan et al. [10] serum sclerostin levels were found to be significantly lower in PsA patients compared with controls. However, in their study most of the patients were controlled on medications including corticosteroids, methotrexate, and anti-TNF, and their mean disease activity scores were much lower than that of our patients.

Sclerostin is a small protein expressed by the SOST gene in osteocytes, bone cells that respond to mechanical stress applied to the skeleton and appear to play an important role in the regulation of bone remodeling. When sclerostin binds to its receptors on the cell surface of osteoblasts, a downstream cascade of intracellular signaling is initiated, with the ultimate effect of inhibiting osteoblastic bone formation. Recent studies have shown that the SOST gene is also expressed by articular chondrocytes and that modulation of its activity may have effects on articular cartilage and subchondral bone. The role of sclerostin in the pathogenesis of osteoarthritis in humans has not yet been defined, and the potential utility of treating osteoarthritis with interventions that alter sclerostin is not known [11].

Rare genetic skeletal disorders in humans with low sclerostin levels, such as sclerosteosis and van Buchem disease, have been associated with a high bone mineral density (BMD) phenotype and low risk of fractures. This has led to the concept that antisclerostin interventions might be useful in the treatment of patients with osteoporosis and skeletal disorders associated with low bone mass. Compounds that inhibit sclerostin have been shown to stimulate bone formation and reduce bone resorption, with a robust increase in BMD. Investigational monoclonal antibodies to sclerostin, including romosozumab, blosozumab, and BPS804, have advanced to phase II clinical trials or beyond. If antisclerostin therapy is found to have beneficial effects on clinical endpoints, such as reduction of fracture risk or improvement in quality of life in patients with osteoarthritis, with a favorable balance of benefit and risk, then this class of compounds may become a prominent addition to the options for therapy of osteoporosis and other skeletal disorders [12].

To the best of our knowledge, this is a novel study to evaluate the validity if sclerostin in prediction of Psoriatic arthritis.

The current study showed that as regard the validity of sclerostin in prediction of cases and it show that at cut off point 2180.65 the sensitivity was 50 with specificity of 60 and accuracy of 47.5 with AUC 0.593. Regarding validity of sclerostin in prediction of Psoriatic arthritis and it show that at cut off point 2113.9 the sensitivity 
was 50 with specificity of 50 and accuracy of 50 with AUC 0.545. Regarding validity of sclerostin in prediction of Psoriasis and it show that at cut off point 2113.9 the sensitivity was 95 with specificity of 50 and accuracy of 72.5 with AUC 0.815 . Regarding validity of sclerostin in prediction of Psoriatic arthritis and psoriasis and it show that at cut off point 1011.55 the sensitivity was 60 with specificity of 40 and accuracy of 50 with AUC 0.42.

Psoriatic arthritis (PsA) is an inflammatory form of arthritis that belongs to the family of spondyloarthritis $(\mathrm{SpA})$ and is related to skin psoriasis. The incidence and prevalence of the disease vary considerably between countries. PsA is classified into axial PsA and peripheral PsA, with a wide range of other extra-articular manifestations. Although the aetiology of the disease is unknown, genetic, environmental, and immunologic factors appear to affect its appearance. In recent years, the role of the immune system in the pathogenesis of PsA has been increasingly investigated. Specific cytokines such as tumor necrosis factor (TNF), interleukin (IL-) 17 and IL-23, play an essential role affecting joint structures. This observation led to the emergence of tumor necrosis factor inhibitors (TNFi) that offer considerable therapeutic benefit to PsA patients. However, chronic inflammation causes bone loss, while new bone formation may also occur in both peripheral and axial skeleton. The molecular mechanisms underlying these processes have not yet been fully understood. So far, the role of the $\mathrm{Wnt} / \beta$-catenin pathway and its inhibitors (Dickkopf and sclerostin) has been evaluated in ankylosing spondylitis (AS), but in PsA has not been studied sufficiently [13].

Sclerostin is a SOST gene product that reduces osteoblastic bone formation by inhibiting canonical $\mathrm{Wnt} / \beta$-catenin signaling. Investigational monoclonal antibodies to sclerostin have been shown to increase bone formation markers and decrease bone resorption markers, with an increase in bone mass [12].

\section{Conclusion}

The significantly higher serum sclerostin levels in PsA patients, compared with controls; there was positive significant correlation between sclerostin and each of age and PASI score. Sclerostin plays an important role in the pathogenesis of PsA and its associated bone damage, either systemic or localized. We can hypothesize that the increased serum sclerostin is a compensatory mechanism to inflammatory process. The use of antisclerostin or other Wnt signaling inhibitors in PsA is still to be investigated because of the risk for increased new bone formation.

\section{References}

[1] R. Warren , A. Menter, Handbook of Psoriasis and Psoriatic Arthritis. Springer; vol. 5(6), pp. 87-90, 2016.

[2] C. H. Smith , "British Association of Dermatologists guidelines for biologic therapy for psoriasis 2017," Br. J. Dermatol; vol. 177(3), pp. 628-636, 2017.

[3] H. Rahimi , C. T. Ritchlin, "Altered bone biology in psoriatic arthritis," Curr. Rheumatol. Rep;vol. 14(4), pp. 349-357, 2012.

[4] P. Rahman , J. T. Elder, "Genetic epidemiology of psoriasis and psoriatic arthritis," Ann. Rheum. Dis; vol. 64(2), pp. 37-39, 2005.

[5] M. Roudier , "Sclerostin is expressed in articular cartilage but loss or inhibition does not affect cartilage remodeling during aging or following mechanical injury," Arthritis Rheum; vol. 65(3), pp. 721-731, 2013.

[6] K. E. S. Poole , "Sclerostin is a delayed secreted product of osteocytes that inhibits bone formation," FASEB J; vol. 19(13), pp. 1842-1844, 2005.

[7] E.-A. A. M. Enas, F. A. Dina, E.-M. E. Reem, S. Y. Dalia, "Study of serum sclerostin levels in association to entheseal ultrasonography in Egyptian psoriatic arthritis patients," Egypt. Rheumatol. Rehabil; vol. 44(2), pp. 45-51, 2017.

[8] S. El-Bakry, N. Saber, H. Zidan, D. Samaha, "Sclerostin as an innovative insight towards understanding rheumatoid arthritis," Egypt. Rheumatol; vol. 38(2), pp. 71-75, 2016.

[9] V. M. Vargas-Muñoz , "Association between sclerostin and bone mineral density in a Mexican sample of women with rheumatoid arthritis: A pilot study," J. Arthritis; vol. 6(1), pp. 1-6, 2015.

[10] E. F. Tarhan , "Sclerostin and Dickkopf-1 but not Periostin may have a role in psoriatic arthritis," ; vol. 2016(5), pp. 764-754, 2016.

[11] M. J. C. Moester, S. E. Papapoulos, C. Löwik, R. L. Van Bezooijen, "Sclerostin: current knowledge and future perspectives," Calcif. Tissue Int; vol. 87(2), pp. 99-107, 2010.

[12] E. M. Lewiecki, "Role of sclerostin in bone and cartilage and its potential as a therapeutic target in bone diseases," Ther. Adv. Musculoskelet. Dis; vol. 6(2), pp. 48-57, 2014.

[13] A. I. Venetsanopoulou , "Clinical, Serological and Immunological Characteristics in Greek Patients with Psoriatic Arthritis: The Role of IL-17, IL-23, and Sclerostin," Mediterr. J. Rheumatol; vol. 31(2), pp. 23-25, 2020. 